\title{
Room Temperature Photonic Crystal Defect Lasers at Near-Infrared Wavelengths in InGaAsP
}

\author{
O. J. Painter, Student Member, OSA, A. Husain, A. Scherer, J. D. O'Brien, Member, OSA, \\ I. Kim, and P. D. Dapkus, Fellow, IEEE
}

\begin{abstract}
Room temperature lasing from optically pumped single defects in a two-dimensional (2-D) photonic bandgap (PBG) crystal is demonstrated. The high- $Q$ optical microcavities are formed by etching a triangular array of air holes into a halfwavelength thick multiquantum-well waveguide. Defects in the 2-D photonic crystal are used to support highly localized optical modes with volumes ranging from 2 to $3(\lambda / 2 n)^{3}$. Lithographic tuning of the air hole radius and the lattice spacing are used to match the cavity wavelength to the quantum-well gain peak, as well as to increase the cavity $Q$. The defect lasers were pumped with 10-30 ns pulses of $0.4-1 \%$ duty cycle. The threshold pump power was $1.5 \mathrm{~mW}(\approx 500 \mu \mathrm{W}$ absorbed $)$.
\end{abstract}

Index Terms - InGaAsP, microcavities, photonic crystals, quantum-well laser, semiconductor device fabrication, spontaneous emission control.

\section{INTRODUCTION}

$\mathbf{T}$ HE proposed use of photonic crystals to modify the optical radiation from emitters within the crystal dates back to the seminal papers of Yablonovitch [1] and John [2]. In this paper we report on the use of a two-dimensional (2D) photonic crystal to localize light to a single defect, thus forming a high- $Q$ microcavity laser with a modal volume less than 0.03 cubic microns. The confinement of the defect mode energy to this tiny volume, and the predicted enhancement of the spontaneous emission rate [3], [4] make the defect cavity a very interesting device for low threshold lasers [5], [6] and high modulation rate light-emitting diodes [7]. Nanooptic structures formed from photonic crystals also hold a great deal of promise due to the flexibility in their geometries. Lithographic methods may be employed to alter the photonic crystal geometry so as to tune device characteristics. An array of densely packed photonic crystal waveguides [8], prisms [9], and light sources [10] integrated on a single monolithic chip may thus be envisioned. Lithographically defined photonic crystal cavities may also find use in some material systems as an alternative to epitaxially grown mirrors, such as for long wavelength vertical-cavity surface-emitting lasers and GaN-based devices.

Manuscript received July 28, 1999; revised August 27, 1999. This work was supported by the Army Research Office under Contracts DAAH04-96-10389 and DAAD19-99-1-0121, and the NSF under Contract ECS-9632937.

O. J. Painter, A. Husain, and A. Scherer are with the Department of Electrical Engineering, California Institute of Technology, Pasadena, CA 91125 USA.

J. D. O'Brien, I. Kim, and P. D. Dapkus are with the Department of Electrical Engineering-Electrophysics, University of Southern California, Los Angeles, CA 90089 USA.

Publisher Item Identifier S 0733-8724(99)09084-2.
The defect cavities studied here utilize a half-wavelength thick high-index membrane to confine the light vertically by way of total internal reflection (TIR) similar to the design of a whispering gallery microdisk laser. The high-index slab is then perforated with a hexagonal array of air holes which Bragg reflects the light in-plane. A defect is formed in the 2D photonic lattice by removing an air hole and/or adjusting the diameters of a few neighboring air holes. A mode, or set of modes depending on the defect geometry, which is highly localized to the defect region is formed. Photons can escape from the defect cavity by tunneling through the 2D photonic crystal, or by leaking out vertically from the waveguide. An illustration of the defect cavity in cross section is shown in Fig. 1. In earlier work [10], we performed an initial demonstration of pulsed lasing action in defect laser cavities where it was necessary to cool the substrate down to $150 \mathrm{~K}$. The original laser cavities suffered from relatively large losses in the vertical direction due to the large holes used to define the photonic crystal [11]. The work presented here consists of measurements of defect cavities in which the hole size has been reduced, improving the quality factor $(Q)$ of the defect modes from 250 to above 500, resulting in room temperature pulsed operation. The paper is separated into four sections: In Section II we describe the fabrication method, in Section III we model the defect cavity using finite-difference time-domain (FDTD) techniques, in Section IV we present the measured lasing characteristics of the defect cavities, and in Section V we provide some conclusions.

\section{FABRICATION}

The defect cavities were fabricated in the InGaAsP-InP material system. The choice of this material system was based upon its relatively slow surface recombination velocity [12] which is important due to the large surface to volume ratio present in the defect cavities. Metalorganic chemical vapor deposition (MOCVD) was used to grow the laser structure on an indium phosphide (InP) substrate. A schematic of the epitaxy is shown in Fig. 2. Optical gain is provided by four $0.85 \%$ compressively strained quaternary quantum-wells (QW's) [13], [14] designed for a peak emission wavelength of $1.55 \mu \mathrm{m}$ at room temperature. The barriers are also quaternary with a room temperature bandgap of $1.22 \mu \mathrm{m}$. Cladding material $(57.5 \mathrm{~nm})$ identical to the barriers is placed on top and bottom of the active region to isolate the QW's further from the surface and to increase the final waveguide thickness. A sacrificial InP (664 nm) layer is grown beneath the QW's 


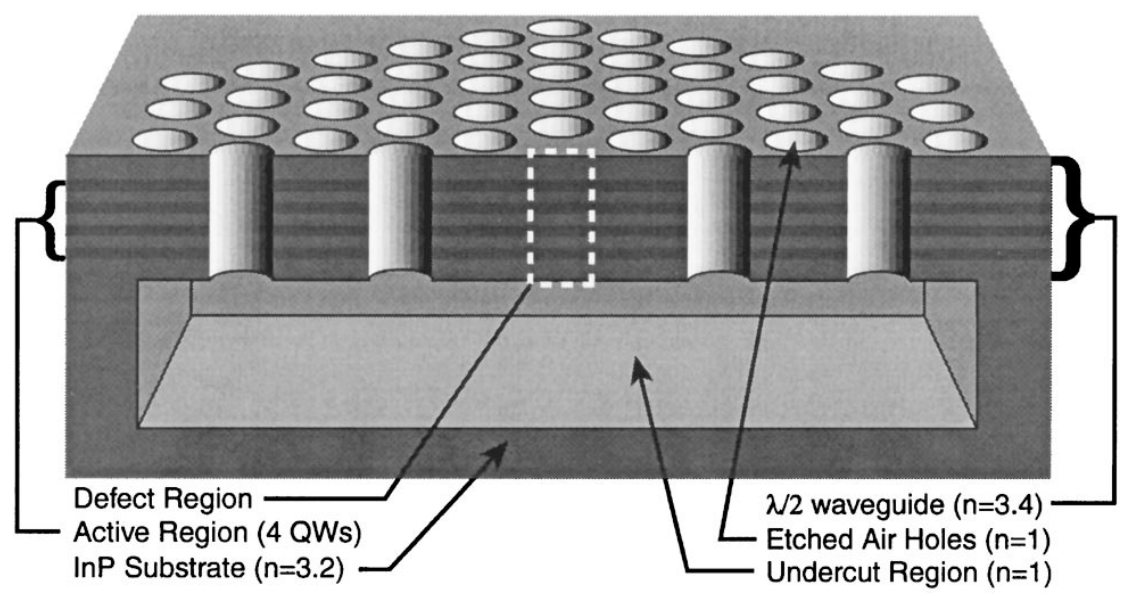

Fig. 1. Illustration of a cross-section through the middle of the photonic crystal microcavity. Photons are localized to the defect region by TIR at the air/slab interface and by Bragg reflection from the 2-D photonic crystal.

\begin{tabular}{|c|c|}
\hline & 50nm InP cap \\
\hline \multirow{4}{*}{$211 \mathrm{~nm}$} & 57.5nm InGaAsP \\
\hline & 9nm InGaAsP QW $(+0.85 \%) \times 4$ \\
\hline & $20 \mathrm{~nm}$ InGaAsP barrier $(\mathrm{Eg}=1.22 \mu \mathrm{m}) \times 3$ \\
\hline & $57.5 \mathrm{~nm}$ InGaAsP $(\mathrm{Eg}=1.22 \mu \mathrm{m})$ \\
\hline \multicolumn{2}{|r|}{$664 \mathrm{~nm}$ InP buffer layer } \\
\hline \multicolumn{2}{|r|}{ 20nm InGaAs etch stop } \\
\hline
\end{tabular}

Fig. 2. InGaAsP-InP epitaxy for the defect lasers. The active region consists of four $0.85 \%$ compressively strained InGaAsP quantum wells. The InP buffer layer is used as a sacrificial layer which is removed by a selective $\mathrm{HCl}$ etch in order to free the membrane from the substrate. The total thickness of the membrane after processing is $211 \mathrm{~nm}$.

and cladding which is subsequently removed in order to free the membrane. A thin InGaAs etch stop layer is grown just below the sacrificial InP layer.

Electron beam lithography is used to first define the photonic crystal pattern in $100 \mathrm{~nm}$ of $2 \%$ polymethyl methacrylate (PMMA). An $\mathrm{Ar}^{+}$ion beam etch is then used to transfer the pattern into an $\mathrm{Au}$ mask layer. This is followed by a $\mathrm{C}_{2} \mathrm{~F}_{6}$ reactive ion etch which etches the holes into a $\mathrm{SiO}_{2}$ layer. The final dry etching step is a $\mathrm{Cl}_{2}$ assisted ion beam etch to transfer the air holes through the active region and into the sacrificial InP layer. Once the dry etching is complete the remains of the surface mask are removed using a buffered oxide etch. The perforated waveguide is then undercut by placing the sample in a slightly agitated $\mathrm{HCl}(4: 1)$ solution at room temperature. The $\mathrm{HCl}$ solution enters through the etched holes and selectively removes the underlying InP material, stopping on the InGaAs etch stop layer, thus providing a smooth bottom interface. Scanning electron microscope (SEM) images of the defect cavity are shown in Figs. 3-5. The resulting membrane is $211 \mathrm{~nm}$ thick and approximately $8 \mu \mathrm{m}$ across. The lattice spacing $(a)$ and the hole radius $(r)$ of the defect cavities studied here are approximately 500 and $160 \mathrm{~nm}$, respectively. At these dimensions a forbidden frequency bandgap opens up for the TE-like (or even) guided modes of the thin membrane which encompasses the emission wavelength of the QW's, as described below.

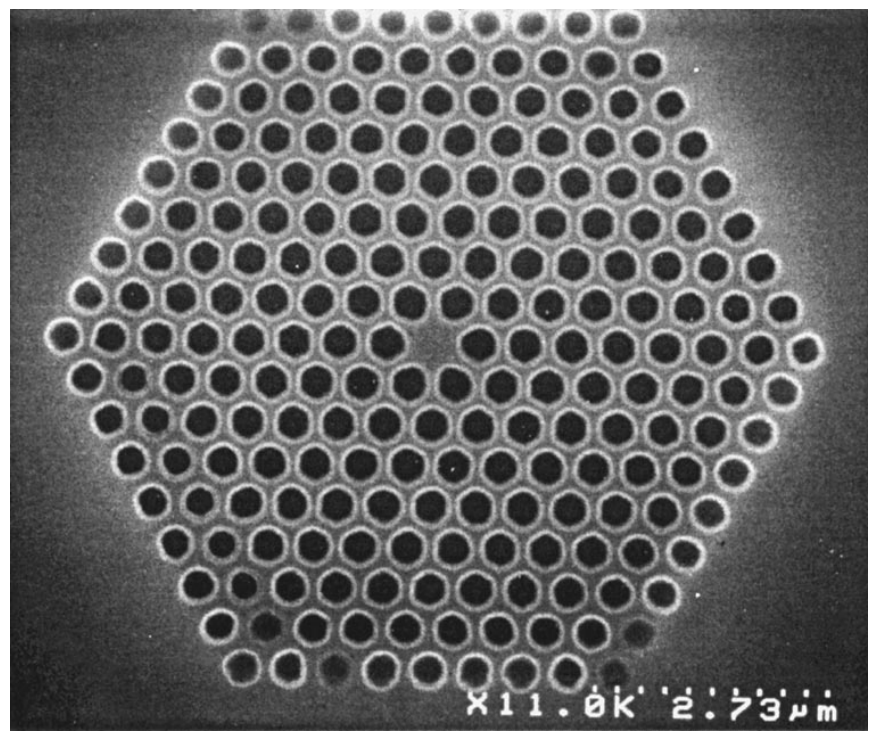

Fig. 3. Top view of a microfabricated 2-D hexagonal array of air holes with a single central hole missing. The interhole spacing, $a$, is $500 \mathrm{~nm}$, and the radius of the holes are approximately $160 \mathrm{~nm}$.

\section{DESIGN}

Three-dimensional (3-D) FDTD simulations [15] can be used to accurately model the electromagnetic modes of a complex structure such as a photonic crystal microcavity. The structure is discretized on a three dimensional mesh and appropriate boundary conditions are applied at the outer surface of the computational domain. In the calculations presented here, an initial electric and magnetic field is defined on the mesh and FDTD is used to step the field in time. The discrete electromagnetic modes of the structure show up as resonance peaks in the Fourier transform of the time-stepped field [16]. A given discrete mode may then be isolated in the structure by convolving the field in time with a bandpass filter. This allows one to associate given mode patterns and symmetries with different resonance peaks. For localized modes of a cavity, the power that is radiated from the computational domain may also be calculated using the Poynting vector, from which one can estimate the quality factor of the mode. In order to elucidate 


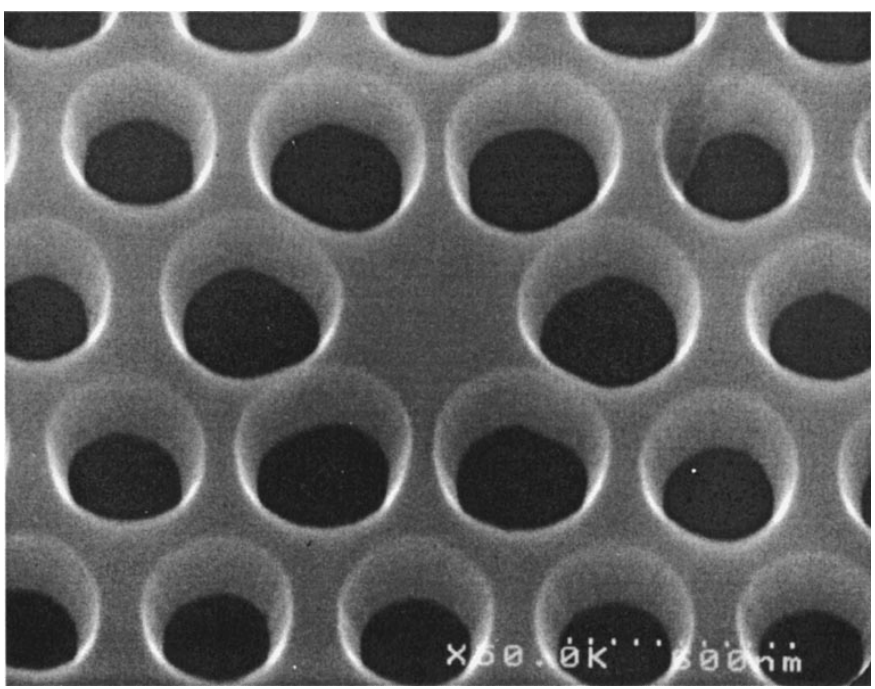

Fig. 4. SEM micrograph of a defect cavity taken at an angle of $30^{\circ}$. After the $\mathrm{HCl}$ etch the etched surfaces are smooth down to a scale from 3 to $5 \mathrm{~nm}$ (SEM resolution). The sidewalls of the holes are sloped at $10^{\circ}$ from vertical, which can be reduced by optimizing the temperature and gas flow during the chemically-assisted ion-beam etch.

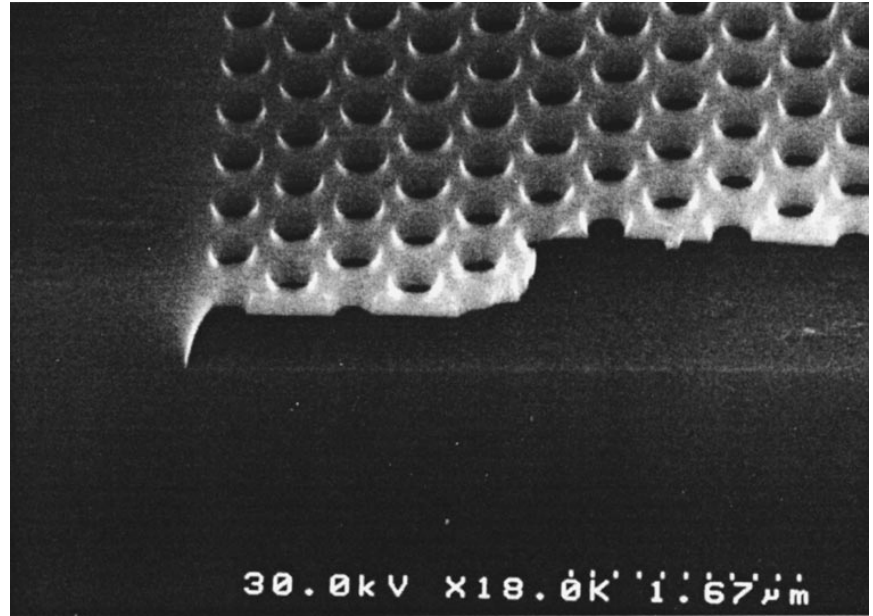

Fig. 5. Cross section through the patterned membrane structure. The slab is $211 \mathrm{~nm}$ thick after processing. The use of an InGaAs etch stop layer results in a smooth bottom interface below the membrane. For membranes larger than $10 \mu \mathrm{m}$ a significant bowing occurs and for devices greater than $15 \mu \mathrm{m}$ the membrane collapses onto the InGaAs etch stop layer.

many of the results discussed in Section IV it will be important to analyze the effects of varying cavity geometries on the defect modes of the structure using these FDTD methods.

The defect microcavity studied here is formed from two basic building blocks: A high-index slab, and a 2-D photonic crystal. Since the high-index slab is approximately a half wavelength thick, it is not accurate to neglect the finite nature of such a 2-D photonic crystal. The guided modes of a symmetric perforated waveguide can be classified simply as even or odd modes, pertaining to the mode symmetry about the horizontal mirror plane in the middle of the slab. The even and odd vertically guided modes may be termed TE-like and TMlike, respectively, in connection to the modes of a 2-D photonic crystal which is infinite in the third direction [17]. The TE-like and TM-like labels only provide an accurate description for

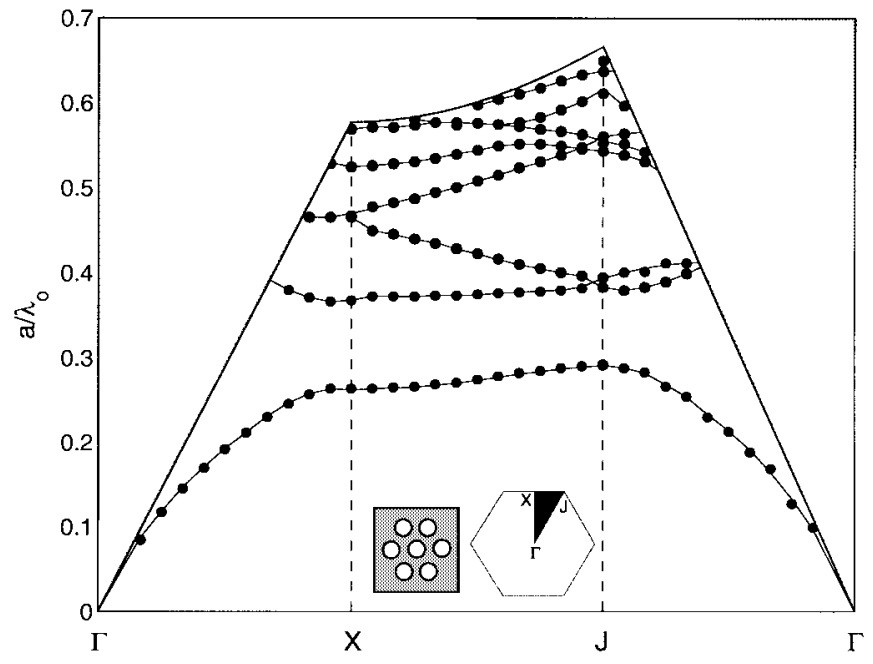

(a)

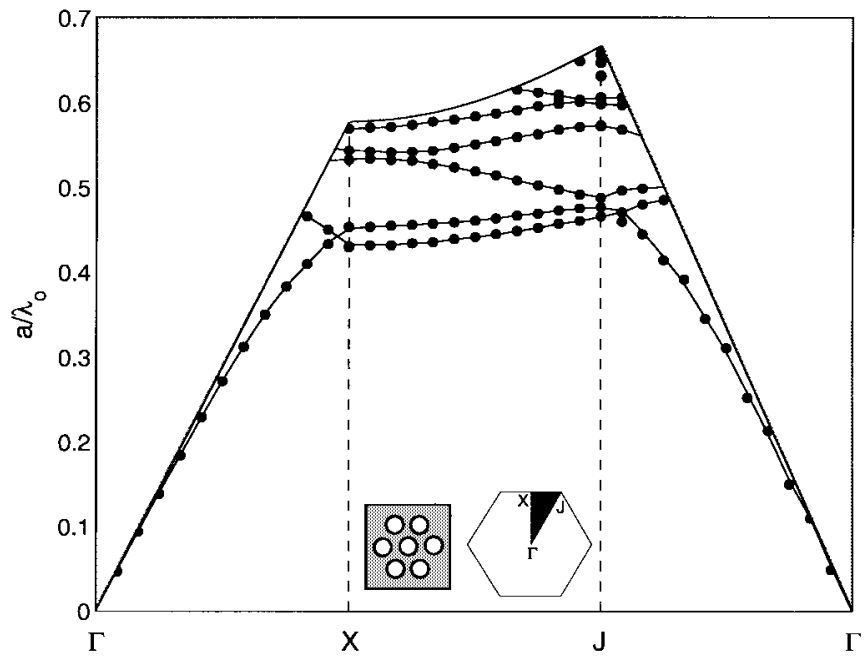

(b)

Fig. 6. In-plane band-structure of the triangular lattice of air holes in an optically thin high-index slab for: (a) the TE-like (even) modes and (b) the TM-like (odd) modes. The parameters used in this calculation are: $r / a=0.32, d / a=0.409, n_{\text {slab }}=3.4$, and $n_{\text {clad }}=1$

the lower lying frequency bands of the fundamental vertically guided modes. A more detailed analysis of the modes of finite 2-D photonic crystal slabs can be found in the work by Russell et al. [18] and Villeneuve et al. [19].

The photonic crystal lattice used in this work is a triangular array of air holes as depicted in the SEM image of Fig. 3. The important parameters in defining the photonic crystal structure are the lattice spacing $(a)$, the hole radius $(r)$, the slab thickness $(d)$, the slab index $\left(n_{\text {slab }}\right)$, and the outer cladding index $\left(n_{\text {clad }}\right)$. A dispersion diagram of frequency versus inplane momentum for the TE-like (even) modes is given in Fig. 6(a). In this calculation $r / a$ was set to $0.32, d / a$ to $0.409, n_{\text {slab }}$ to 3.4 , and $n_{\text {clad }}$ to 1 . This corresponds to the mean value of the parameters for the fabricated devices studied in Section IV. The shaded region in Fig. 6(a) and (b) is for frequencies above the light line of photons in the air cladding. Photons with frequencies above the light line can propagate in the air, and thus are leaky resonant modes of the slab or 
nonresonant radiation modes. The bands plotted below the light line represent the guided modes of the photonic crystal slab. As shown in Fig. 6(a) there exists a frequency bandgap for the transverse electric (TE)-like guided modes between the first two bands. We will use the terms "conduction" and "valence" band to describe the upper and lower bands defining the bandgap, respectively. The valence band is completely guided over the entire first Brillouin zone (IBZ), while the conduction band is leaky around the $\Gamma$ point. The leaky nature of the conduction band is the fundamental source of vertical radiation loss from the defect cavity. The band diagram of the transverse magnetic (TM)-like (odd) guided modes is plotted in Fig. 6(b). With an $r / a$ ratio of 0.32 and a slab refractive index of 3.4 a bandgap does not form for the TM-like guided modes. For our purposes, however, this is beneficial as it will limit the number of high- $Q$ localized modes of the defect (TE-like only).

Lateral confinement of the optical field is obtained by removing a single hole in the photonic lattice creating a local potential energy well for photons. For the index contrasts studied here $(3.4: 1)$ a pair of localized dipole-like modes form which are very similar in nature to the defect modes of the infinite 2-D photonic crystal [17]. The localized modes of this cavity can be described as a linear combination of the $x$ and $y$ dipole modes shown in Fig. 7. The electric field of the diopole modes is polarized predominantly in the plane of the slab and has an antinode in the center of the defect thus providing good overlap with the active material. The defect modes of this structure have been previously analyzed in detail elsewhere [11], [19]. Using the relation [20]

$$
V_{\text {mode }}^{\text {eff }} \equiv \frac{\int_{V} \epsilon(\vec{r})|\vec{E}(\vec{r})|^{2} d^{3} r}{\max \left(\epsilon(\vec{r})|\vec{E}(\vec{r})|^{2}\right)}
$$

the mode volume of the dipole modes is estimated to be 2.5 $(\lambda / 2 n)^{3}$, which is roughly 0.03 cubic microns at a wavelength of $1.55 \mu \mathrm{m}$.

As the dimensions of a laser cavity are reduced, the feedback from the laser mirrors must also increase in order to maintain the photon lifetime in the cavity $\left(t_{p h}\right)$. For the defect cavity which is almost a half-wavelength on a side it is critical that the power loss from the 2-D photonic crystal mirrors is minimized. The quality factor $(Q)$ of the localized modes in the defect cavity can be calculated from the stored energy $(U)$ and radiated power $\left(P_{\mathrm{rad}}\right)$ as follows:

$$
Q \equiv \omega \frac{U(t)}{P_{\text {rad }}(t)}
$$

where $\omega$ is the angular frequency of the mode. The power radiated from the defect can be divided into a component due to photons which leak vertically from the waveguide, and a component due to photons which tunnel through the finite number of periods of the photonic crystal. The energy which escapes by tunneling can be reduced by simply adding more periods to the photonic crystal (limited eventually by scattering or absorption). The power which leaks out of the waveguide vertically can not be captured by adding more periods of the photonic crystal, and thus poses a much more serious limitation

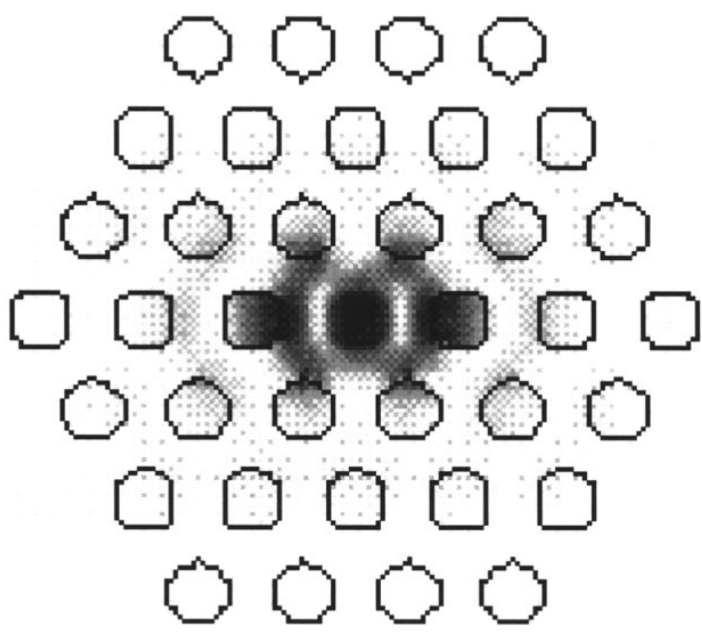

(a)

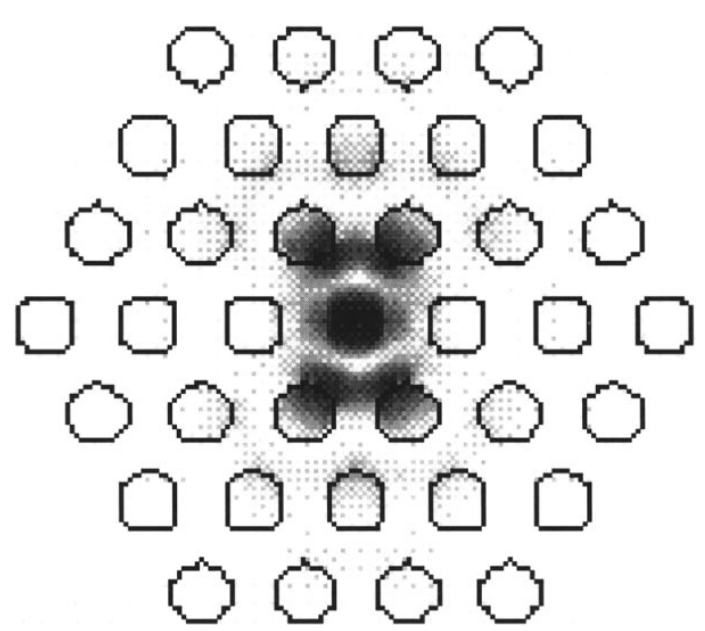

(b)

Fig. 7. Two-dimensional slice through the middle of the membrane showing the electric field amplitude of the degenerate dipole modes: (a) the $x$-dipole mode and (b) the $y$-dipole.

on the cavity $Q$. In order to reduce the vertical loss smaller air holes are preferred, which both increases the effective index guiding of the slab (less material removed) and reduces the photonic bandgap (PBG). The smaller bandgap results in a slightly shallower energy well, resulting in a mode which is broader in real space but narrower in $k$-space. The narrowing in $k$-space of the defect mode to regions around the bandedge ( $X$-point) reduces the coupling to leaky portions of the conduction band in Fig. 6. The calculated $Q$ value for the cavities analyzed in the next section varies between 500-600 $(r / a \approx 0.31$ and $d / a \approx 0.41)$, an improvement by a factor of two relative to the earlier low temperature lasers. The $Q$ value can be increased further by increasing the waveguide thickness or by increasing the lateral dimension of the cavity [11], [19], however this is at the expense of increasing the mode volume and potentially increasing the number of localized cavity modes.

\section{Measurement Results and Discussion}

A large array of defect cavities was fabricated with varying lattice spacings and hole sizes in order to cover a wide range 
of wavelengths of the defect mode resonance. It was found that for a lattice spacing of $525 \mathrm{~nm}$ and a hole radius of 165 $\mathrm{nm}$ the cavity mode resonance matched the gain peak of the multiquantum well active region. The defect cavities consist of eight periods of the photonic crystal surrounding a single removed hole in the center. With eight periods of photonic crystal the defect mode theoretically emits most strongly in the vertical direction thus allowing measurements to be taken from the top of the sample.

The sample was mounted on an $\mathrm{X}-\mathrm{Y}-\mathrm{Z}$ stage and the defect cavities were optically pumped from above at an angle normal to the sample surface. A high numerical aperture, long working distance $100 \times$ objective lens was used to both image the defect cavities and to focus the optical pump beam. An 830-nm semiconductor laser diode was used as the pump source in this experiment. The photoluminescence (PL) was also collected by the objective lens and then fed into an optical spectrum analyzer. A GaAs filter was placed just before the optical spectrum analyzer to separate the pump from PL. The spot size of the beam on the sample surface could be adjusted down to $1 \mu \mathrm{m}$, however a spot size of approximately $4 \mu \mathrm{m}$ was found to be optimum in the sense of providing the lowest threshold for lasing. We attribute this to the fact that the QW's in the photonic crystal mirror surrounding the defect will be absorbing if not pumped to transparency. Absorption in the 2-D photonic crystal mirror will then limit the $Q$ of the defect cavity. FDTD calculations [11] indicate that to obtain a $Q$ of roughly 200 requires at least three periods of the photonic lattice surrounding the defect, which corresponds to a 3- $\mu \mathrm{m}$ diameter. As the pump is defocused to increase the spot size above $4 \mu \mathrm{m}$ the intensity in the center of the cavity decreases to a point where the threshold pump power starts to rise. For consistency a pump beam spot size of roughly $4 \mu \mathrm{m}$ was used throughout to take the data.

With the pump beam focused to a $4-\mu \mathrm{m}$ spot, we first measured the PL from an unprocessed area on the sample very near to the array of defect cavities. In Fig. 8 the PL spectra is shown for various duty cycles and pump powers. At lowpump powers (bottom plot) the emission is peaked at 1545 $\mathrm{nm}$ which corresponds to the first energy level in the QW's. As the pump power is increased (middle plot) a peak in the emission spectrum appears at $1380 \mathrm{~nm}$ corresponding to the second level in the QW's. The top plot was obtained using similar pumping conditions as those used to obtain lasing in the defect cavities (10 ns pulses, $0.3 \%$ duty cycle). The PL is very broad in this case, providing almost a $400 \mathrm{~nm}$ wide emission range over which the photonic crystal cavities can be characterized.

The PL from the defect cavities is markedly different than from the unprocessed areas. The emission spectrum for various pump powers from a defect cavity with a lattice spacing of 525 $\mathrm{nm}$ is shown in Fig. 9. The emission can be seen to be strongly frustrated except for two peaks. The broad shorter wavelength peak at $1425 \mathrm{~nm}$ most likely corresponds to spontaneous emission from the conduction band of the photonic crystal. The narrow longer wavelength peak at $1580 \mathrm{~nm}$ is the defect mode laser line. In Fig. 10, we plot the theoretical resonances of the defect cavity as calculated by FDTD along with the
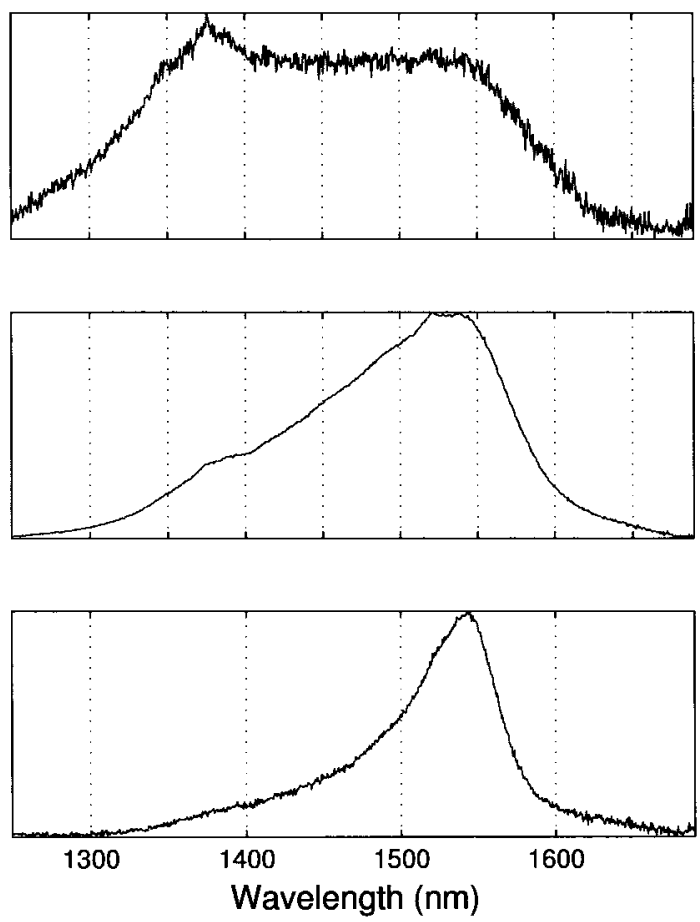

Fig. 8. PL from an unprocessed area is shown for three different pumping conditions (4- $\mu \mathrm{m}$ pump spot size). The bottom plot corresponds to $20 \mu \mathrm{W}$ continuous wave $(\mathrm{CW})$ pumping. In the middle plot the peak pump power is $170 \mu \mathrm{W}$ with a $50 \%$ duty cycle. The PL in the top plot was taken using $10 \mathrm{~ns}$ pulses with a $3-\mu$ s period at a peak pump power of $7 \mathrm{~mW}$ (duty cycle $\sim 0.3 \%$ ).

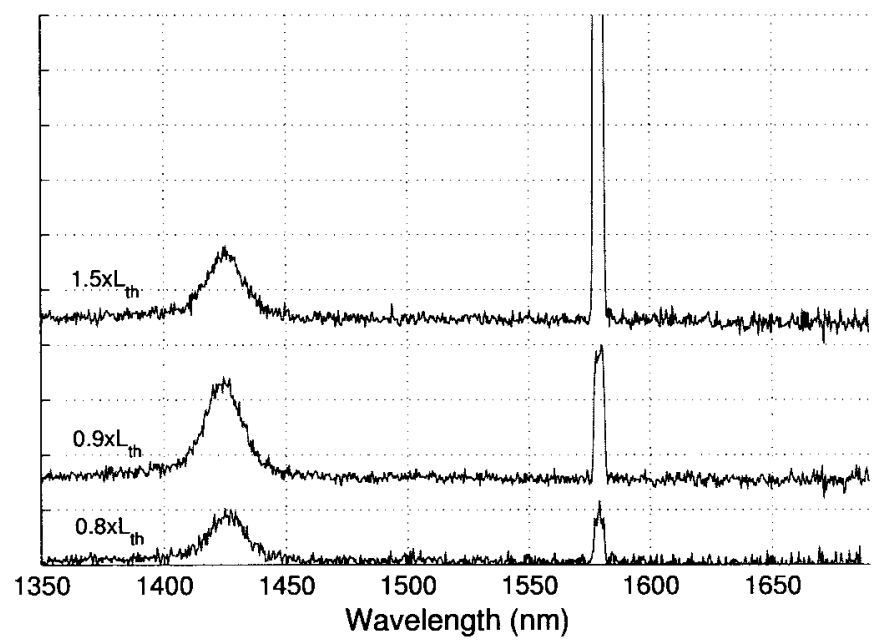

Fig. 9. PL spectra from a defect cavity with $a=525 \mathrm{~nm}$ and $r / a=0.32$. The pump power is increased from just below threshold (bottom) to 1.5 times threshold (top) in the series of plots.

experimentally measured PL. The vertical dashed lines in Fig. 10 represent the extent of the calculated TE-like guided mode bandgap. The defect laser line is seen to match up very well with the calculated defect mode resonance. The spontaneous emission peak at $1425 \mathrm{~nm}(a / \lambda=0.37)$ also matches up very nicely with the photonic crystal conduction band-edge. Although the modes at the conduction band-edge are guided in an ideal slab (Fig. 6), the sidewalls supporting the membrane (Fig. 5) may scatter the light into the collection lens. 


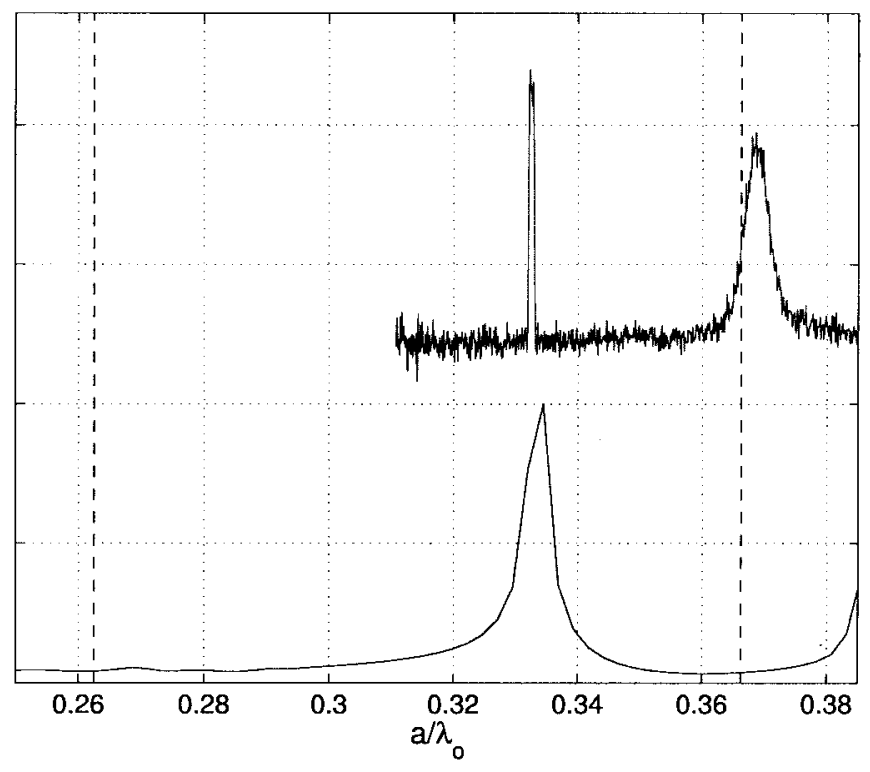

Fig. 10. Plot of the experimentally measured PL (top) along with an FDTD simulation of the defect cavity (bottom). The defect cavity in both the simulation and experiment had a lattice spacing of $525 \mathrm{~nm}$ and $r / a=0.32$. The band edges of the TE-like guided mode bandgap are shown as vertical dashed lines.

A plot of the collected power versus the pump power $(L-L)$ for a typical defect cavity is shown in Fig. 11. The laser remains single mode over the entire pumping range of this plot. The external threshold pump power (peak) is approximately $1.5 \mathrm{~mW}$, from which we estimate an absorbed power of $500 \mu \mathrm{W}\left(\sim 3.1 \mathrm{~kW} / \mathrm{cm}^{2}\right)$. The laser line becomes linearly polarized (measured in the plane of the slab) above threshold. The fiber coupled average power from some of the defect laser cavities is as high as $4 \mathrm{nW}$, which corresponds to an approximate peak power of $1 \mu \mathrm{W}$. This is roughly $30 \mathrm{~dB}$ above the shorter wavelength resonance peak of the conduction band. We were able to obtain lasing at duty cycles up to $1 \%$ (30 ns pulses) however threshold pump powers were significantly increased due to heating in the membrane. The relatively large threshold powers are most likely a result of the poor heat sinking of the membrane cavity. Nonradiative Auger recombination exponentially increases with temperature and is especially problematic in small bandgap materials such as InGaAsP [21].

The linewidth of the defect mode narrows from $1.1 \mathrm{~nm}$ just below threshold to $0.15 \mathrm{~nm}$ (resolution limited) at twice threshold. An estimate of the $Q$ of the defect modes can be made by measuring the linewidth of the resonance at transparency. From an approximate absorbed threshold pump power of $500 \mu \mathrm{W}$ we estimate the threshold carrier density is 3 times the transparency carrier density. At a duty cycle of $0.3 \%$ the average detected power was too small to make accurate linewidth measurements near the transparency pumping level. Due to the temperature sensitivity of the membrane cavities we were also not able to increase the duty cycle much beyond $1 \%$ without significantly reducing the PL due to nonradiative effects. For several of the higher threshold devices linewidths of $\sim 2.5 \mathrm{~nm}$ were measured at pump levels which were 2.5 times below threshold. This gives an upper bound $Q$ value of

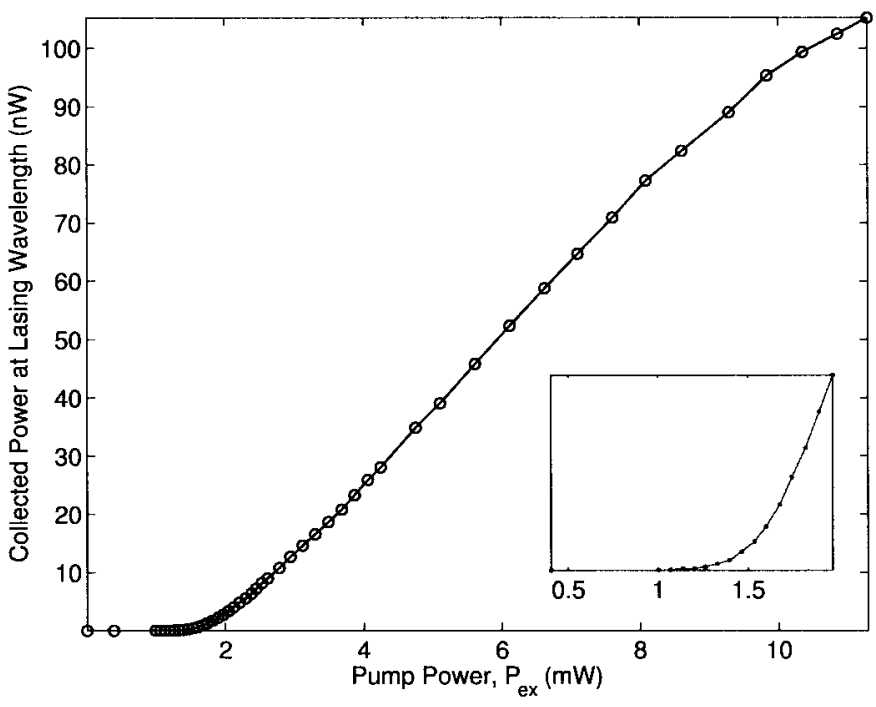

Fig. 11. Plot of the collected power at the lasing wavelength versus input pump power (peak external value).

600 for the higher threshold defect modes which is consistent with the FDTD calculated values.

\section{SUMMARY}

In conclusion, we have demonstrated pulsed room temperature lasing at $1.5 \mu \mathrm{m}$ in an optically pumped 2-D photonic crystal cavity with a single missing hole (defect). The modal volume of the defect modes is theoretically as small as 2.5 $(\lambda / 2 n)^{3}$. By reducing the hole size of the 2-D photonic crystal mirror we have been able to reduce the waveguide losses of the defect cavity and increase the $Q$ by a factor of two or more. The estimated absorbed threshold pump power of the defect cavities is $500 \mu \mathrm{W}$, limited mainly by nonradiative Auger recombination due to heating of the membrane cavity. FDTD simulations of the defect cavity agree very well with the measured photoluminescence data and are used to design such parameters as lasing wavelength, emission pattern, and cavity $Q$.

\section{ACKNOWLEDGMENT}

O. J. Painter would like to thank K. Vahala and T. Yoshie for many helpful discussions and R. Johnson for his help in setting up the experimental apparatus.

\section{REFERENCES}

[1] E. Yablonovitch, "Inhibited spontaneous emission in solid-state physics and electronics," Phys. Rev. Lett., vol. 58, no. 20, pp. 2059-2062, May 1987.

[2] S. John, "Strong localization of photons in certain disordered dielectric superlattices," Phys. Rev. Lett., vol. 58, no. 23, pp. 2486-2489, June 1987.

[3] E. M. Purcell, "Spontaneous emission probabilities at radio frequencies," Phys. Rev., vol. 69, p. 681, 1946.

[4] R. Coccioloi, M. Boroditsky, K. W. Kim, Y. Rahmat-Samii, and E Yablonovitch, "Smallest possible electromagnetic mode volume in a dielectric cavity," Inst. Elec. Eng. Proc.—Optoelectron, Dec. 1998, vol. 145, no. 6, pp. 391-397.

[5] H. Yokoyama, "Physics and device application of optical microcavities," Science, vol. 256, pp. 66-70, Apr. 1992. 
[6] Y. Yamamoto and S. Machida, "Microcavity semiconductor laser with enhanced spontaneous emission," Phys. Rev. A, vol. 44, no. 1, pp. 657-668, July 1991.

[7] L. A. Graham, D. L. Huffaker, and D. G. Deppe, "Spontaneous lifetime control in a native-oxide-apertured microcavity," Appl. Phys. Lett., vol. 74, no. 17, pp. 2408-2410, Apr. 1999.

[8] S.-Y. Lin, E. Chow, V. Hietala, P. R. Villeneuve, and J. D. Joannopoulos, "Experimental demonstration of guiding and bending of electromagnetic waves in a photonic crystal," Science, vol. 282, pp. 274-276, Oct. 1998.

[9] H. Kosaka, T. Kawashima, A. Tomita, M. Notomi, T. Tamamura, T Sato, and S. Kawakami, "Superprism phenomena in photonic crystals," Phys. Rev. B, vol. 58, no. 16, pp. 10 096-10099, Oct. 1998.

[10] O. Painter, R. K. Lee, A. Yariv, A. Scherer, J. D. O’Brien, P. D. Dapkus, and I. Kim, "Two-dimensional photonic bandgap defect mode laser," Science, vol. 284, pp. 1819-1824, June 1999.

[11] O. Painter, J. Vučković, and A. Scherer, "Defect modes of a twodimensional photonic crystal in an optically thin dielectric slab," J. Opt. Soc. Amer. B, vol. 16, no. 2, pp. 275-285, Feb. 1999.

[12] L. A. Coldren and S. W. Corzine, Diode Lasers and Photonic Integrated Circuits. New York: Wiley, 1995.

[13] I. Kim, D. G. Chang, and P. D. Dapkus, "Growth of InGaAsP in a stagnation flow vertical reactor using TBA and TBP," J. Cryst. Growth, vol. 119, no. 1-4, pp. 138-143, Dec. 1988

[14] Y. Zou, J. S. Osinski, P. Grodzinski, P. D. Dapkus, W. Rideout, W. F. Sharfim, and F. D. Crawford, "Experimental verification of strain benefits in $1.5 \mu \mathrm{m}$ semiconductor lasers by carrier lifetime and gain measurements," IEEE Photon. Technol. Lett., vol. 4, no. 12, pp. 1315-1318, Dec. 1992

[15] K. S. Yee, "Numerical solution of boundary value problems involving Maxwell's equations in isotropic media," IEEE Trans. Antennas Propagat., vol. 14, pp. 302-307, 1966.

[16] C. T. Chan, Q. L. Yu, and K. M. Ho, "Order-N spectral method for electromagnetic waves," Phys. Rev. B, vol. 51, no. 23, pp. 16635-16642, June 1995.

[17] J. D. Joannopoulos, R. D. Meade, and J. N. Winn, Photonic Crystals. Princeton, NJ: Princeton University Press, 1995.

[18] P. St. J. Russell, D. M. Atkin, and T. A. Birks, Bound Modes of TwoDimensional Photonic Crystal Waveguides. Amsterdam, The Netherlands: Kluwer Academic, 1996, pp. 203-218.

[19] P. R. Villeneuve, S. Fan, S. G. Johnson, and J. D. Joannopoulos, "Threedimensional photon confinement in photonic crystals of low-dimensional periodicity," in Inst. Elec. Eng. Proc.-Optoelectron., Dec. 1998, vol 145 , no. 6, pp. 384-390.
[20] J. S. Foresi, P. R. Villeneuve, J. Ferrera, E. R. Thoen, G. Steinmeyer, S. Fan, J. D. Joannopoulos, L. C. Kimerling, H. I. Smith, and E. P. Ippen, "Photonic-bandgap microcavities in optical waveguides," Nature, vol. 390, pp. 143-145, Nov. 1997.

[21] G. P. Agrawal and N. K. Dutta, Semiconductor Lasers. New York: Van Nostrand Reinhold, 1993.

O. J. Painter, photograph and biography not available at the time of publication.

A. Husain, photograph and biography not available at the time of publication

A. Scherer, photograph and biography not available at the time of publication

J. D. O'Brien, photograph and biography not available at the time of publication.

I. Kim, photograph and biography not available at the time of publication.

P. D. Dapkus (F'87), photograph and biography not available at the time of publication. 